\title{
Frontières
}

\section{Maladie d'Alzheimer et droits des personnes en fin de vie dans les législations européennes}

\section{Federico Palermiti}

Volume 20, numéro 1, automne 2007

La « bonne mort »

URI : https://id.erudit.org/iderudit/017954ar

DOI : https://doi.org/10.7202/017954ar

Aller au sommaire du numéro

Éditeur(s)

Université du Québec à Montréal

ISSN

1180-3479 (imprimé)

1916-0976 (numérique)

Découvrir la revue

Citer cet article

Palermiti, F. (2007). Maladie d'Alzheimer et droits des personnes en fin de vie dans les législations européennes. Frontières, 20(1), 89-92.

https://doi.org/10.7202/017954ar
Résumé de l'article

Cet article examine la problématique particulière qui se pose pour la personne atteinte de la maladie d'Alzheimer, pour ses proches et pour ses soignants relativement aux directives anticipées sur l'intensité des soins en fin de vie. Différentes législations européennes qui encadrent l'expression préalable de la volonté de la personne en fin de vie sont brièvement présentées ainsi que les modalités de désignation d'un tiers pour exprimer cette volonté. Quelques exemples, choisis en Europe, d'approches spécifiquement adaptées aux personnes atteintes de troubles cognitifs sont discutées, en distinguant les approches conceptuelles et les approches pragmatiques. 


\section{Résumé}

Cet article examine la problématique particulière qui se pose pour la personne atteinte de la maladie d'Alzheimer, pour ses proches et pour ses soignants relativement aux directives anticipées sur l'intensité des soins en fin de vie. Différentes législations européennes qui encadrent l'expression préalable de la volonté de la personne en fin de vie sont brièvement présentées ainsi que les modalités de désignation d'un tiers pour exprimer cette volonté. Quelques exemples, choisis en Europe, d'approches spécifiquement adaptées aux personnes atteintes de troubles cognitifs sont discutées, en distinguant les approches conceptuelles et les approches pragmatiques.

Mots clés: directives anticipées - soins de fin de vie - maladie d'Alzheimerlégislations européennes.

\begin{abstract}
This article discusses the particular problem that arises for people with Alzheimer's disease, for their family and for their caregivers concerning advance directives on the intensity of care at the end-of-life. Various European legislation surrounding the prior expression of end-of-life persons' wishes are briefly presented as are the procedures for appointing a third party to express this will. Some examples chosen in Europe of approaches specifically adapted for people with cognitive disorders are discussed, distinguishing conceptual and pragmatic approaches.
\end{abstract}

Keywords: advance directives end-of-life care - Alzheimer's disease European legislation.

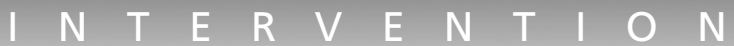

\section{Maladie d'Alzheimer et droits des personnes en fin de vie dans les législations européennes}

\section{Federico Palermiti, juriste, chargé d'études, \\ Fondation Médéric Alzheimer ${ }^{1}$, Paris, France. palermiti@med-alz.org}

Parce que ses implications médicales, sociales, économiques et juridiques s'avèrent particulièrement complexes, la maladie d'Alzheimer oblige nos sociétés à repenser la place de l'usager dans les dispositifs d'accompagnement et de soin. Au fur et à mesure de l'évolution de la maladie, et plus particulièrement en fin de vie, les fonctions cognitives de la personne malade s'altèrent progressivement. Comme nous le rappelle Mara, 57 ans, atteinte de la maladie d'Alzheimer, la faculté de discernement et la capacité à exprimer ses souhaits peuvent s'avérer diminuées: «ma crainte, ce n'est pas de mourir, c'est d'avoir peut-être une infime part de "moi", du vrai moi, qui survive tout en étant incapable de communiquer les craintes et les souhaits les plus élémentaires ${ }^{2} »$. Anticiper ces situations de fin de vie complexes semble alors être une des réponses émergentes dans un certain nombre de législations en Europe. En effet, ces législations tendent à reconnaître progressivement la faculté pour une personne de porter à la connaissance des équipes de soins ses souhaits quant à sa fin de vie, pour le jour où elle sera devenue incapable de les exprimer. Ainsi toute personne majeure, juridiquement capable, peut communiquer ses décisions de fin de vie, soit directement, en rédigeant une directive anticipée, soit indirectement, par l'intermédiaire d'un tiers qui la représentera. Reste que des divergences apparaissent encore en Europe quant à la valeur juridique de ces deux mesures et à leur prise en compte par les équipes de soins. Certains pays européens n'ont d'ailleurs toujours pas reconnu de statut juridique à ce type de dispositifs ${ }^{3}$. Enfin, leur mise en œuvre, dans le cas des personnes atteintes de la maladie d'Alzheimer, n'est pas sans soulever un certain nombre de questions juridiques et éthiques. C'est pourquoi il nous est apparu intéressant de proposer une sélection d'approches en Europe spécifiquement adaptées aux personnes atteintes de troubles cognitifs.

\section{L'EXPRESSION PRÉALABLE DE LA VOLONTÉ DE LA PERSONNE EN FIN DE VIE}

En rédigeant une directive anticipée, toute personne peut indiquer, par avance, ses volontés quant aux décisions relatives à sa fin de vie pour le jour où elle ne sera plus 


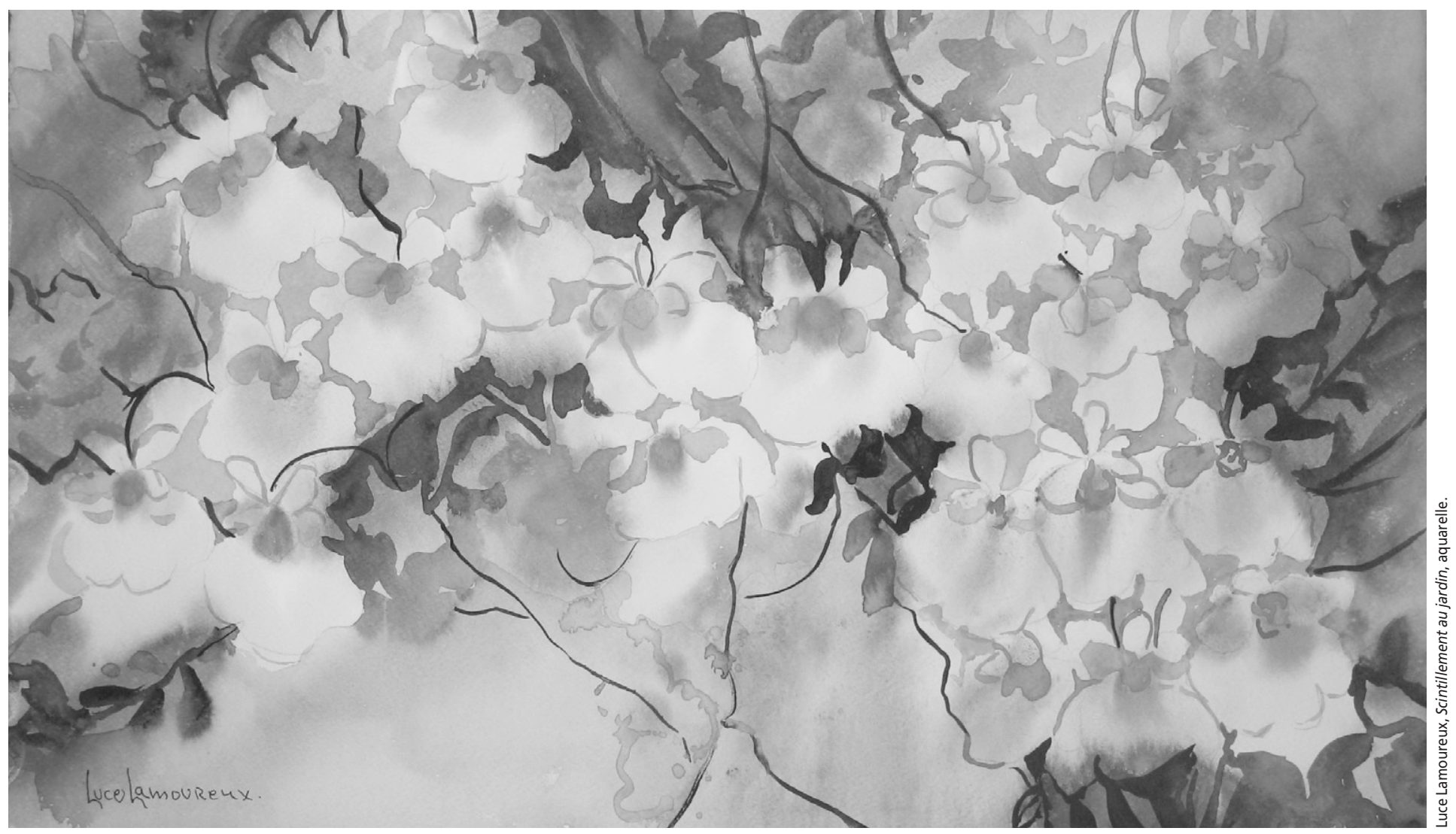

en mesure de les exprimer. Dans l'ensemble des législations européennes étudiées ${ }^{4}$, les règles liées au formalisme, à la durée et au contenu des directives anticipées s'avèrent être relativement homogènes. En effet, se présentant principalement sous la forme d'un document écrit, signé et daté par la personne, les directives anticipées peuvent également consister en une fiche pré-imprimée remplie et signée par la personne avec le médecin devant témoins. Les décisions médicales susceptibles d'être couvertes par les directives anticipées s'avèrent rarement limitées, tout comme leur durée de validité$^{5}$. Enfin, la possibilité pour la personne malade de modifier ou révoquer ses souhaits anticipés est également envisagée par l'ensemble des pays. Concernant la portée des instructions contenues dans une directive anticipée, tantôt ces dernières sont juridiquement contraignantes, comme en Belgique, au Royaume-Uni et en Espagne (bien que pour ce dernier pays, l'autonomie des différentes régions en matière médicale hypothèque la valeur reconnue aux directives anticipées d'une région à l'autre); tantôt leur portée est absolue uniquement lorsque la personne malade est en fin de vie (Allemagne) ou en cas de phase terminale (Danemark). D'autres pays ne leur reconnaissent qu'une simple valeur. En effet, en France ${ }^{6}$, par exemple, les directives anticipées ne s'imposent pas au médecin. Le législateur français a toutefois prévu, dans le cas où la personne se trouve hors d'état d'exprimer sa volonté, la mise en œuvre d'une procédure collégiale dans laquelle la prise en compte des volontés de la personne malade est impérative, et ce, préalablement à la prise de décision par le médecin de limitation ou d'arrêt du traitement.

Dans la mesure où les directives anticipées ne sont entachées d'aucune illégalité de fond (incapacité de la personne, contrainte, etc.) ou de forme, qu'en est-il de la mise en œuvre des instructions qu'elles contiennent? Trois questions semblent conditionner la prise en compte par les équipes médicales des instructions inscrites dans les directives anticipées, et ce, indépendamment de leur valeur juridique (contraignante ou non).

- Les instructions contenues dans la directive anticipée sont-elles suffisamment claires et détaillées? En effet, plus ces instructions seront précises et complètes, mieux les équipes médicales seront en mesure de les appliquer. À cet égard, les efforts de mise à disposition d'informations et d'aide détaillées pour les personnes et les professionnels concernant la rédaction des directives anticipées sont très variables d'un pays à l'autre.

- Ces instructions correspondent-elles raisonnablement à la situation évoquée? Une des limites majeures de la mise en œuvre des instructions contenues dans une directive anticipée tient effectivement au fait que l'évolution de la maladie s'avère difficilement prévisible, tout comme «les options thérapeutiques [... qui] peuvent évoluer entre le moment de la rédaction de la directive et celui de sa mise en pratique» (Berghmans et Widdershoven, 2005, p. 153).

- Ces instructions sont-elles contraires à la loi ou la bonne pratique médicale? S'opposeraient ainsi à l'application d'une directive anticipée une demande d'euthanasie faite par la personne dans les pays n'ayant pas légiféré en la matière ou des instructions jugées contraires à l'«intérêt du patient».

En ce qui concerne les personnes atteintes de la maladie d'Alzheimer, un tel dispositif soulève, par ailleurs, un certain nombre d'interrogations juridiques et éthiques. En effet, au cours de l'évolution de la maladie et plus particulièrement à un stade avancé, comment s'assurer que les souhaits antérieurement exprimés par la personne malade correspondent toujours à ses désirs actuels? Quelle valeur juridique les équipes médicales doiventelles accorder à des souhaits nouvellement exprimés, dans la mesure où les directives anticipées sont révocables et révisables à tout moment? Plus généralement, la complexité et la spécificité de la fin de vie dans la maladie d'Alzheimer rendent les options thérapeutiques difficilement prévisibles: un temps de la fin de vie plus long que pour 
certaines maladies terminales, des prises de décisions complexes (nutrition, douleur, acharnement/abandon thérapeutique...), une communication du bien-être et du mal-être possible jusqu'en phase terminale (Killick et Allan, 2006), mais nécessitant une formation des équipes de soins. Enfin, la stigmatisation de la maladie et la peur qu'elle suscite peuvent avoir comme conséquence que les directives anticipées expriment, bien avant la phase terminale, le désir de ne plus continuer à vivre. La personne malade envisage ainsi sa fin de vie comme une dégradation et une souffrance insupportables alors que, dans de bonnes conditions, ce vécu peut s'avérer être tout autre: "beaucoup de personnes souffrent de la peur que représente la perspective de la maladie et non de la maladie elle-même ${ }^{7}$ ».

Il n'en demeure pas moins que, de l'avis des soignants, les directives anticipées représentent un moyen d'aborder la question de la fin de vie au moment où la personne est encore en mesure d'exprimer des choix. De même, lorsque la période de la fin de la vie s'installe, les directives anticipées peuvent constituer un instrument de référence pour entrer en relation avec la personne malade et chercher à appréhender ses souhaits actuels (Berghmans et Widdershoven, 2005). D'un point de vue juridique, les directives anticipées, notamment pour les personnes atteintes de troubles cognitifs, constituent, si elles sont rédigées suffisamment tôt, l'un des éléments permettant d'assurer le plus longtemps possible l'autonomie des personnes. La désignation par la personne malade d'un tiers «mandataire de santé » pour exprimer ses souhaits vient, par ailleurs, compléter ce dispositif.

\section{LA DÉSIGNATION D'UN TIERS POUR EXPRIMER LA VOLONTÉ DE LA PERSONNE EN FIN DE VIE}

Les proches, parce qu'ils sont liés à la personne malade par des liens familiaux, d'affection ou tutélaires, ont toujours joui d'une légitimité de fait en matière de consentement aux soins. Récemment, un certain nombre de législations en Europe se sont dotées de dispositions qui reconnaissent la représentation en matière médicale. Il convient toutefois de distinguer ce tiers «mandataire de santé » et le tiers intervenant au titre du régime de protection des majeurs juridiquement incapables (tuteur, curateur, etc.) qui peut intervenir, selon les législations considérées, en matière de consentement ou de refus de soins ${ }^{8}$. Cette «représentation» en matière médicale constitue une véritable avancée puisqu'il appartient à la personne malade elle-même de désigner un tiers supposé rendre compte de ses volontés lorsqu'elle sera devenue incapable de les exprimer. Reste que, dans les pays européens étudiés, les contours de cette "représentation» sont fonction à la fois des systèmes juridiques dans lesquels elle s'insère et de l'autonomie plus ou moins grande que l'on souhaite reconnaître au «mandataire». En France, depuis 2002, il est possible pour la personne malade de désigner une "personne de confiance», pouvant être un parent, un proche ou le médecin traitant, laquelle sera consultée si la personne malade se trouve être hors d'état d'exprimer sa volonté ou de recevoir l'information requise 9 . En Angleterre et au pays de Galles, c'est une récente loi globale sur la capacité des personnes qui a étendu à l'actuel attorney une compétence sur les questions de santé et de prise en charge médicale ${ }^{10}$. En Écosse, l'Adults with Incapacity Act de 2000 autorise le mandat en matière médicale (proxy). En Espagne, notamment en cas d'absence de directives anticipées, il existe la possibilité de désigner, par un mandat de représentation, une «personne de confiance» ou un membre de la famille en vue de faire respecter ses dernières volontés, notamment à propos des soins de fin de vie. Le Danemark reconnaît également aux proches ou au tuteur l'autorité et le pouvoir pour donner un consentement en matière médicale.

Qu'en est-il de l'étendue de cette représentation instituée? Quelle portée juridique revêtent les choix exprimés par le «mandataire de santé »? En France, la personne de confiance ne possède pas de réel pouvoir de décision. Tout au plus est-elle «la voix» de la personne malade. La législation ne lui accordant qu'un rôle consultatif dans la procédure collégiale, la décision d'arrêt ou de poursuite d'un traitement appartient en dernier ressort au médecin ${ }^{11}$. En Allemagne, l'avis du «mandataire» désigné pour prendre les décisions médicales doit être confirmé par un tribunal si la décision risque de causer un préjudice important au patient, voire de provoquer son décès. En Angleterre et au pays de Galles, la loi de 2005 retient la même solution. En Écosse, en dépit du véritable progrès que représente la loi de 2000 , le recours à la notion d'«intérêt du patient» hypothèque le pouvoir de «représentation du mandataire». En cas de conflit ou de désaccord entre le médecin et le «mandataire », un deuxième médecin doit donner un nouvel avis sur le traitement engagé. C'est également le recours à la notion d' "intérêt du patient» qui, en Espagne, permet aux médecins de trancher le conflit pouvant naître entre le «mandataire de santé» et toute autre personne (Berrocal Lanzarot, 2005).
En pratique, le statut et le rôle du «mandataire de santé » sont encore peu connus des professionnels et des personnes malades elles-mêmes. La reconnaissance de un ou plusieurs interlocuteurs privilégiés dans les prises de décision se limite souvent encore pour les professionnels à l'entourage familial identifié. Or, la faculté pour la personne malade de désigner un tiers en dehors du cadre familial ordinaire pourrait favoriser une certaine «neutralité » dans les décisions que ce dernier prendra.

Rappelons, enfin, que dans le cadre de la maladie d'Alzheimer et des maladies apparentées, les personnes malades concernées par la question de la représentation en matière médicale sont généralement des personnes âgées et les conséquences liées à l'avancée en âge, tant familiales (perte du conjoint, absence des enfants) que sociales (isolement), limitent la mise en œuvre d'un tel dispositif. Aussi, un certain nombre d'entre elles ne désigne pas de tiers pouvant rendre compte de leurs volontés.

\section{APPROCHES SPÉCIFIQUES POUR LES PERSONNES ATTEINTES DE LA MALADIE D'ALZHEIMER: QUELQUES EXEMPLES EN EUROPE}

Parce que les législations n'ont pas vocation à dire "ce qu'il faut faire [en fin de vie], mais [à fixer] le cadre dans lequel les décisions doivent être prises» (Leonetti, 2006), il nous est paru intéressant de sélectionner quelques exemples d'approches en Europe, spécifiquement adaptées aux personnes atteintes de troubles cognitifs.

\section{Approches conceptuelles}

D'un point de vue juridique et afin de préserver le plus longtemps possible l'autonomie et la capacité juridique de la personne malade, l'exemple de la loi écossaise ${ }^{12}$ permet de s'interroger sur la notion même de capacité juridique en proposant, en matière de décision médicale, que la capacité de la personne malade perdure dans le plus grand nombre d'hypothèses possibles. Cette capacité est appréciée en fonction du domaine de prise de décision. Si cette approche s'inscrit bien dans une démarche déontologique classique, elle constitue, en s'inscrivant dans un cadre juridique, une véritable avancée des représentations concernant les compétences décisionnelles des personnes malades. Concernant les prises de décisions de fin de vie en établissement, les équipes de soins peuvent se trouver démunies face à des situations difficiles, aux enjeux éthiques complexes (hydratation, nutrition, abandon thérapeutique...). Les équipes inventent alors des réponses plus ou moins structurées pour avancer dans leur réflexion et dans leur prise de déci- 
sions (groupes de parole, groupes de prise de décision, atelier éthique, centre d'éthique...). Formaliser les processus de prise de décision par la mise en place de comités d'éthique internes à l'établissement semble également être une réponse adaptée à la spécificité de la fin de vie dans la maladie d'Alzheimer (Grob et Schmieder, 2006). Ainsi, ce comité d'éthique aurait pour mission de prendre les décisions de fin de vie complexes (notamment en faisant appel à un spécialiste de «l'éthique») et d'assurer un suivi de la décision prise collégialement.

\section{Approches pragmatiques}

La désignation d'un professionnel en charge de conseiller les personnes malades et leurs aidants sur ces dispositifs juridiques (médecin-traitant, case manager) et le développement d'outils d'information pour les personnes et les professionnels du soin constitue un préalable nécessaire pour la mise en œuvre effective des droits des personnes atteintes de la maladie d'Alzheimer' ${ }^{13}$.

Concernant les personnes malades sans proches, sans entourage, n'ayant désigné aucune personne de confiance, n'ayant rédigé aucune directive anticipée et n'étant pas encore sous un régime de protection, la question de leur représentation reste cruciale. À ce titre, l'exemple des Independent Mental Capacity Advocate en Angleterre s'avère particulièrement pertinent, dans la mesure où est instauré un mécanisme de «représentation institutionnelle» pour ces personnes. Leur mission consiste principalement à défendre, représenter et accompagner les personnes malades tout au long de leur maladie.

Ainsi, dans une très grande majorité des pays européens étudiés, les dispositions qui encadrent juridiquement les directives anticipées ou la «représentation» en matière médicale constituent une avancée significative en faveur de la préservation de l'autonomie de la personne malade, même lorsqu'elle est devenue incapable d'exprimer sa volonté. Rappelons que, dans la maladie d'Alzheimer, ces mesures ne s'avèrent effectives qu'au seul stade précoce de la maladie et que le développement d'outils facilitant leur mise en œuvre compléterait le dispositif actuel. Toutefois, à travers ces deux mesures, le dialogue entre soignants et personnes malades tend vers davantage de réciprocité: certains n'hésitent d'ailleurs pas à parler désormais de "décision partagée » (Camhi et Bremond, 2004) en lieu et place de la traditionnelle notion de «consentement aux soins». Le manque de recul sur l'application de ces législations doit rester, néanmoins, à l'esprit de tous, au même titre que le risque toujours présent créé par des lois ayant pour objet de statuer sur ces situations limites: en effet, le type de décision dont il est question (arrêt ou abstention de thérapeutiques, respect d'un refus de soins) relève du cas par cas. Ainsi, parce $q u$ '«aucune fin de vie ne ressemble à une autre» (Leonetti, 2006), la réflexion sur les règles éthiques et déontologiques régissant les situations de fin de vie demeure indispensable pour étayer les choix qui seront faits.

\section{Bibliographie}

ALZHEIMER EUROPE (2006). «The use of advance directives by people with dementia», $<$ www.alzheimer-europe.org $>$.

BERGHMANS, R. L. P. et Guy A. M. WIDDERSHOVEN (2005). "Questions d'éthiques: capacité mentale et directives anticipées », Les Cahiers de la Fondation Médéric Alzheimer (édition française), $\mathrm{n}^{\circ} 1$, p. 147-157.

BERROCAL LANZAROT, A. I. (2005). "Analisis de los criterios juridicos en la normativa estatal y autonomica sobre cuidados paliativos e instrucciones previas - el papel del medico en su aplicacion », XII Congreso Nacional de Derecho sanitario, <www.aeds. org $>$.

CAMHI, B. et A. BREMOND (2004). «La décision partagée en droit français: enjeux juridiques de la recherche des préférences des patients lors de la prise de décision médicale».

GROB H. et M. SCHMIEDER (2006). «L'important c'est d'être présent », Les Cahiers de la Fondation Médéric Alzheimer (édition française), $\mathrm{n}^{\circ} 2$, p. 124-132.

KILLICK J. et K. ALLAN (2006). «Le Good Sunset Project: communiquer au seuil de la mort», dans Les Cahiers de la Fondation Médéric Alzheimer (édition française), $\mathrm{n}^{\circ} 2$, p. $145-151$.

LEONETTI, J. (2006). «Une loi qui dit non pas ce qu'il faut faire, mais le cadre dans lequel les décisions doivent être prises ", Les Cahiers de la Fondation Médéric Alzheimer (édition française), n 2 , p. 38-43.

PALERMITI, F. et I. GRANDGÉRARD (2006), «Droits des personnes en fin de vie dans les législations européennes: quelle place pour les personnes atteintes d'altérations cognitives progressives », Les Cahiers de la Fondation Médéric Alzheimer, $\mathrm{n}^{\circ} 2$ (édition française et anglaise).

WORTMANN, M. (2005). Loi sur l'euthanasie et démence aux Pays-Bas, Conférence d'Alzheimer Disease International, Istanbul, $<$ www.alz.co.uk/adi/conference/conf2005. html>.

\section{Notes}

1. Créée en 1999, la Fondation Médéric Alzheimer, reconnue d'utilité publique, s'est donné pour mission d'accroître les connaissances sur les conséquences sociales de la maladie
d'Alzheimer ou de maladies apparentées, de favoriser le développement d'initiatives locales d'aide aux personnes malades et à leurs aidants et de susciter une réflexion sur la prise en charge de la maladie dépassant les clivages entre sciences humaines et sciences médicales.

$<w w w . f o n d a t i o n-m e d e r i c-a l z h e i m e r . o r g>$

2. Témoignages des membres du réseau international de soutien et de défense des droits des personnes atteintes de démence Dementia Advocacy and Support Network International, dans Les Cahiers de la Fondation Médéric Alzheimer, vol. 2, p. 24-29 (édition française).

3. Notamment la Grèce, le Portugal, la Roumanie, la Hongrie, comme le souligne l'Association Alzheimer Europe (voir Alzheimer Europe, 2006)

4. Angleterre et pays de Galles (Mental Capacity Act de 2005 entrée en vigueur en 2007), Belgique (loi du 22 août 2002 relative aux droits des patients), Danemark (loi du $1^{\text {er }}$ juillet 1998 relative au statut des patients), Écosse ("Adults with Incapacity Act» de 2000), Espagne (loi du 14 novembre 2002 relative à l'autonomie du patient et aux droits et obligations en matière d'information médicale), France (loi du 4 mars 2002 relative aux droits des malades et à la modernisation du système de santé et loi du 22 avril 2005 relative aux droits des malades et à la fin de vie), Pays-Bas (loi du 12 avril 2001 relative au contrôle de l'interruption de vie pratiquée sur demande et de l'aide au suicide). Pratique jurisprudentielle en Allemagne et en Italie.

5. À l'exception de la France (article L. 1111-4 du Code de santé publique) où la durée de validité des directives anticipées est fixée à trois ans, renouvelables.

6. La loi du 22 avril 2005 relative aux droits des personnes et à la fin de vie.

7. Wortmann (2005) rappelle également qu'«au Pays-Bas, la pratique de l'euthanasie est légale et en dix ans il y a eu quatre cas d'euthanasie de personnes souffrant de troubles cognitifs demandant que l'euthanasie les soulage de leurs souffrances ».

8. La récente loi française réformant la protection juridique des majeurs vulnérables du 7 mars 2007 a étendu le champ d'intervention du tiers ayant autorité de tutelle: ce dernier est à présent compétent aussi bien pour les affaires patrimoniales que pour les questions de droits des personnes, incluant la santé.

9. Loi du 4 mars 2002 relative aux droits des malades et à la modernisation du système de santé.

10. Mental Capacity Act de 2005, précitée.

11. Loi du 22 avril 2005 relative aux droits des personnes et à la fin de vie.

12. Adults with Incapacity Act, précitée.

13. En Allemagne, un document complet d'information détaillant de manière précise les situations de fin de vie et les instructions susceptibles d'être contenues dans une directive anticipée a été rédigé pour les personnes malades et les professionnels. 